\title{
The treatment of immature heterotopic ossification in spinal cord injury with combination surgery, radiation therapy and NSAID
}

\author{
TM Freebourn ${ }^{1,2}$, DB Barber ${ }^{1,2}$ and AC Able ${ }^{1,2}$ \\ ${ }^{1}$ Spinal Cord Injury Center, South Texas Veterans Health Care System and ${ }^{2}$ Department of Rehabilitation Medicine, \\ The University of Texas Health Science Center at San Antonio, Texas, USA
}

\begin{abstract}
Heterotopic ossification (HO) is a frequent complication associated with spinal cord injury. Management of $\mathrm{HO}$ consists of a combination of range-of-motion, diphosphonates, nonsteroidal antiinflammatory agents, radiation therapy, and in some cases, surgical resection. The appropriate timing of surgical resection has traditionally been based on maturity of the HO. The case presented is that of a 33-year-old male with T8 complete paraplegia who developed $\mathrm{HO}$ about the left hip resulting in impaired sitting. The patient underwent successful surgical wedge resection of the $\mathrm{HO}$ despite apparent immaturity of the HO. A comprehensive review of the literature is presented which suggests that early resection of immature $\mathrm{HO}$ may not be predictive of a higher recurrence rate.
\end{abstract}

Keywords: heterotopic ossification; spinal cord injury; nonsteroidal antiinflammatory agents; radiation therapy

\section{Introduction}

Heterotopic ossification (HO), the development of bone in periarticular soft tissue, was first described in spinal cord injury (SCI) by Dejerine and Ceillier in 1918. ${ }^{1}$ The incidence of HO in SCI is reported to range from $10-52 \% .^{1-11}$ The hallmark of medical management of HO in SCI has consisted of a combination of range-of-motion to prevent ankylosis and either a diphosphonate or nonsteroidal antiinflammatory agent (NSAID) to minimize the extent of ossification. Despite treatment, the incidence of bony ankylosis is $5 \%$ and approximately $20 \%$ of SCI patients will experience some functional loss. ${ }^{4,12}$ Surgical resection of $\mathrm{HO}$ is undertaken in those patients in whom medical management has failed, resulting in a loss of function. Surgery is typically combined with either radiation therapy, a disphosphonate or NSAID, or both. The appropriate timing of surgical intervention has traditionally been based upon confirmation that the $\mathrm{HO}$ is mature, as evidenced by bone scan, in order to reduce the risk of recurrence. Classically, the maturity of $\mathrm{HO}$ in SCI is felt to occur at 12 to 18 months after clinical presentation. ${ }^{3-5,11,12}$ Herein, we report the case of successful treatment of immature $\mathrm{HO}$ via the combination of surgical resection, radiation therapy, and a NSAID.

Correspondence: DB Barber, Department of Rehabilitation Medicine, 7703 Floyd Curl Drive, San Antonio, Texas 782847798, USA

Presented at the Poster Session of the 24th Annual Scientific Meeting of the American Spinal Injury Association, Cleveland, Ohio, April 1998

\section{Case Report}

The patient, a 33-year-old white male with a 9 month history of T8 complete thoracic paraplegic secondary to a pedestrian-motor vehicle accident, presented to an outpatient spinal cord injury clinic for a new patient assessment. His chief complaint was that of continued impairment in his self care and mobility. He attributed this impairment to a restriction in flexion of his left hip that resulted from HO. HO had been diagnosed at approximately 3 months postinjury and was treated with a course of both etidronate disodium and ibuprofen. On physical examination, hip flexion was limited to $75^{\circ}$ by a palpable bony mass (normal $120^{\circ}$ ) (Figure 1). Laboratory evaluation was unremarkable except for an elevated alkaline phosphatase of 147 (normal 39-117) and elevated erythrocyte sedimentation rate (ESR) of 23 (normal <15). 99m-Tc-MDP bone scan documented continued increased uptake in the $\mathrm{HO}$ extending from the left iliac fossa to proximal femur (Figure 2). At 11 months after the diagnosis of $\mathrm{HO}$, the patient underwent wedge resection of that portion of the HO residing at the level of the acetabulum (Figure 3). On postoperative day one, the patient received $700 \mathrm{cGy}$ of radiation to the surgical site. By postoperative day seven, the surgical site was noted to be warm, erythematous, and edematous. Although feeling well, the patient was experiencing fevers up to $103^{\circ} \mathrm{F}$. Laboratory evaluation was unremarkable except for an alkaline phosphatase of 158, ESR of 98, and a normal white blood cell count (WBC) of 5.9 but with 13 bands (normal $0-10$ ). Blood cultures were negative. Repeat $99 \mathrm{~m}-\mathrm{Tc}-\mathrm{MDP}$ bone scan noted partial resection of the $\mathrm{HO}$ and was otherwise 
unchanged from the previous study (Figure 4). The clinical and laboratory findings were felt to be consistent with an acute inflammatory response resulting from a combination of the excision of active $\mathrm{HO}$ and radiation.

The surgical site did not appear infected nor the patient septic. On postoperative day seven, the patient was prescribed indomethacin $25 \mathrm{mg}$ tid for 6 weeks. The fever abated within $48 \mathrm{~h}$ after the initiation of indomethacin and the inflammatory signs were

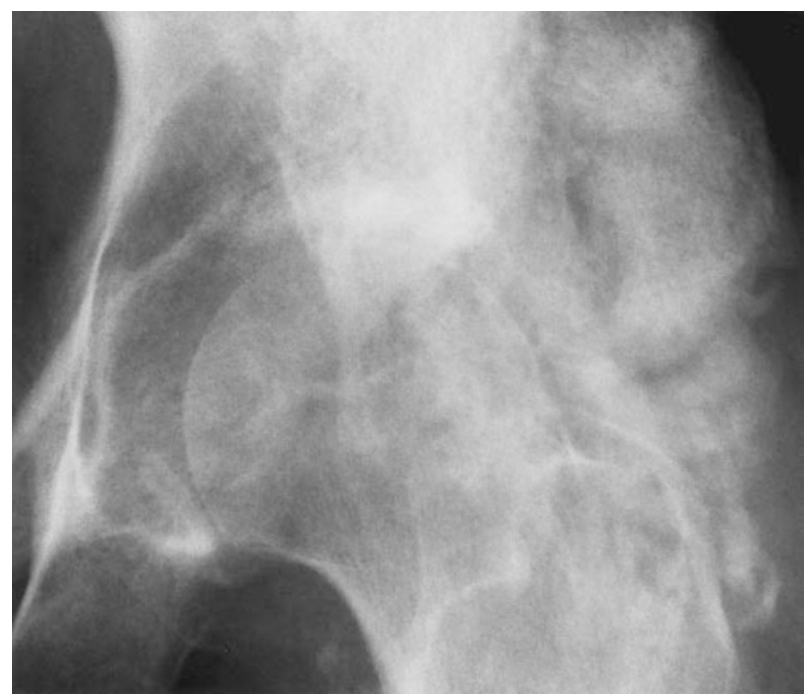

Figure 1 AP of left hip demonstrating heterotopic ossification

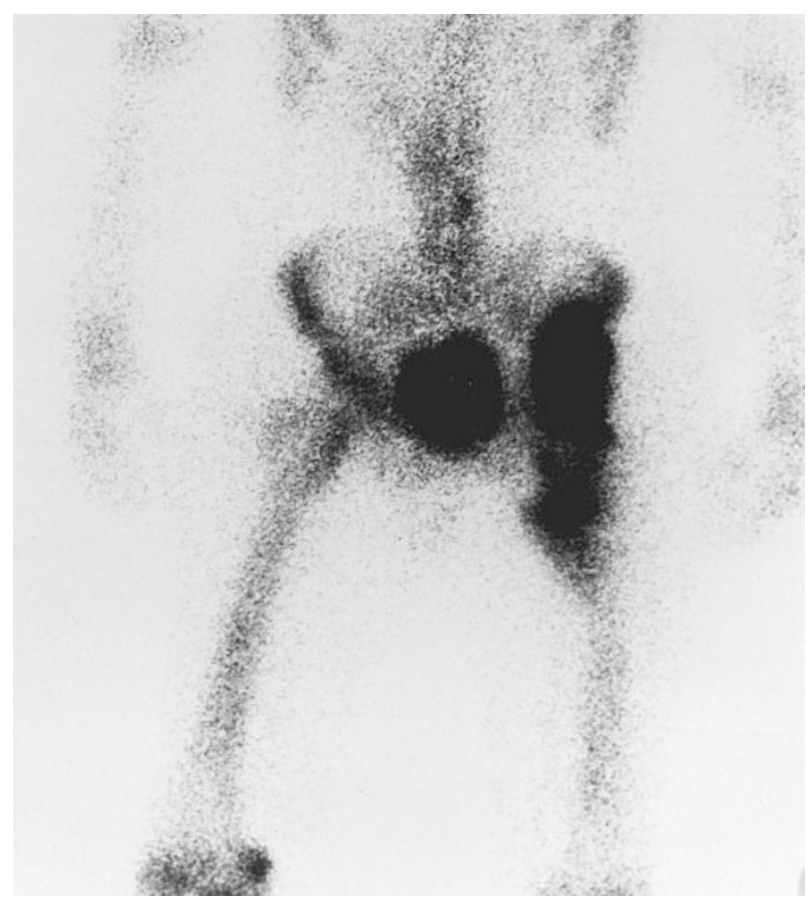

Figure 2 99m-Tc-MDP bone scan demonstrating immature heterotopic ossification about the left hip completely resolved after 3 weeks of indomethacin. At 18 months after surgery, the patient has $110^{\circ}$ of flexion at the hip and is completely independent in his self care and mobility. Radiographically, the excision site is noted to be free of $\mathrm{HO}$ (Figure 5).

\section{Discussion}

$\mathrm{HO}$ in SCI is most frequently diagnosed at between 1 to 4 months postinjury with a peak incidence at 2

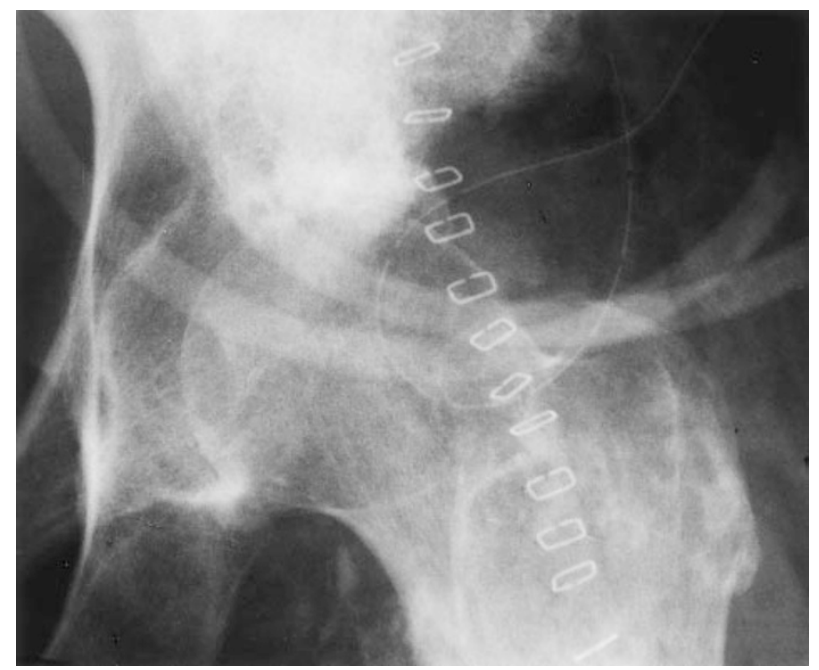

Figure 3 Postoperative AP of left hip demonstrating resection of heterotopic ossification

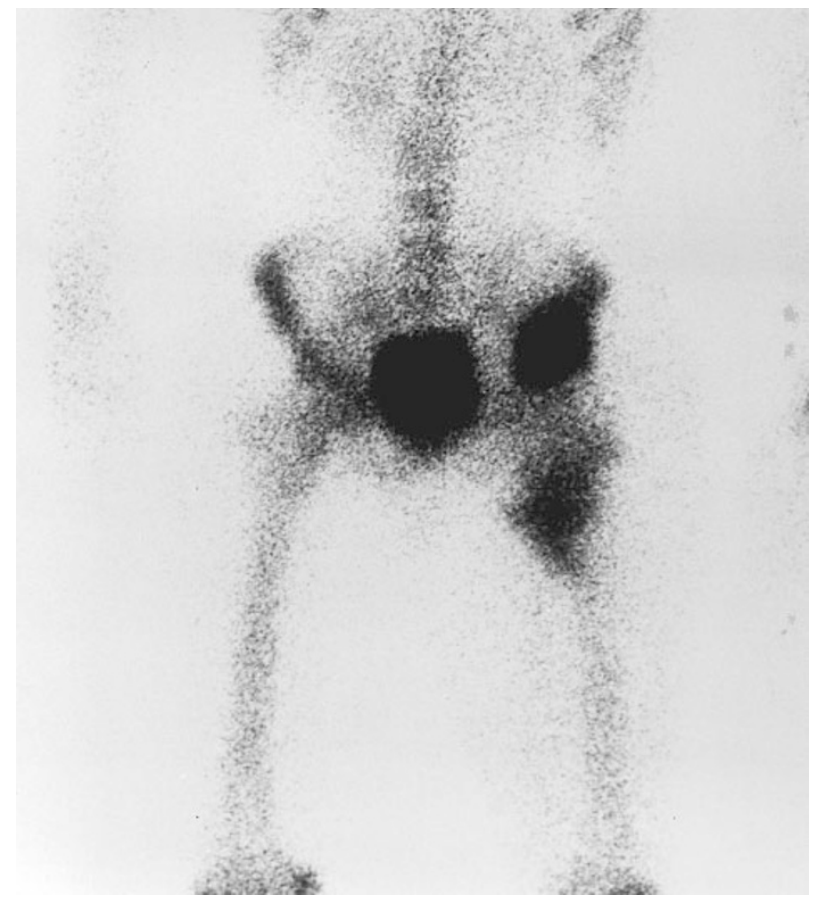

Figure 4 99m-Tc-MDP bone scan demonstrating wedge resection of heterotopic ossification 


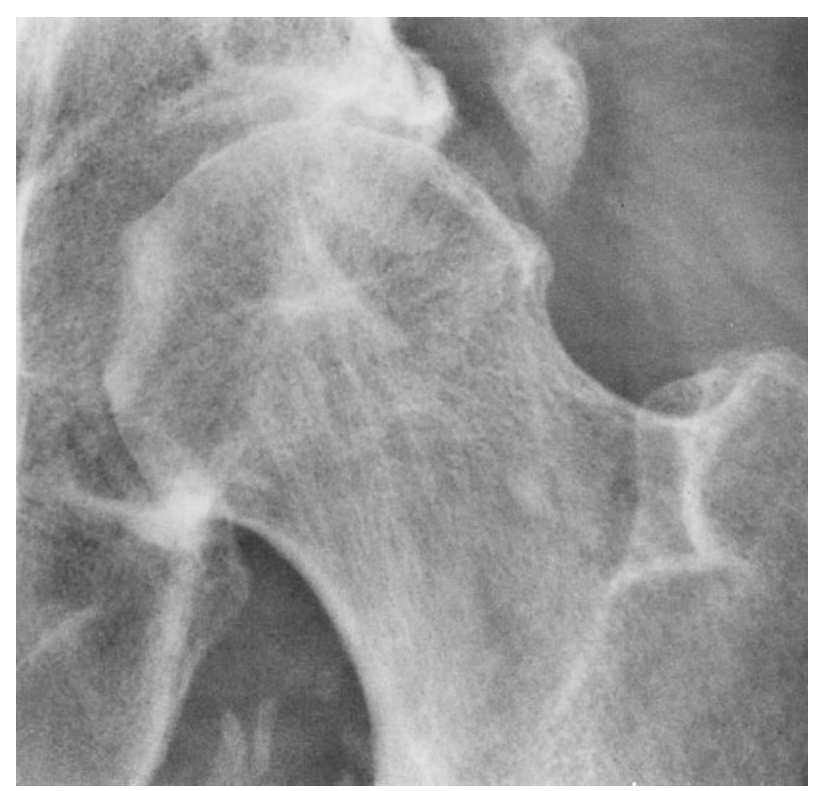

Figure 5 AP of left hip demonstrating long-term success of resection of heterotopic ossification

months. ${ }^{5,7,8,13}$ HO develops below the level of the neurologic injury, most commonly affecting the hip. ${ }^{8,14,15}$ Although first described by Dejerine and Ceillier in 1918, the etiology and pathophysiology of $\mathrm{HO}$ in SCI remains unknown.

Clinical signs and symptoms of HO in SCI include pain, restricted joint range-of-motion, inflammatory signs of warmth, erythema and edema, or the development of pressure sores over the underlying HO. $99 \mathrm{~m}-\mathrm{Tc}-\mathrm{MDP}$ bone scan is felt to be the most sensitive instrument for diagnosing early $\mathrm{HO}$ and assessing HO maturity. ${ }^{13,16-18}$ Plain roentgenograms typically reveal a flocculent, patchy pattern of soft tissue ossification 7 to 10 days after clinical symptoms arise. Although commonly elevated in $\mathrm{HO}$, alkaline phosphatase levels are not specific to HO in SCI. ${ }^{17,18}$

A variety of treatment options have been described for the medical management of $\mathrm{HO}$ in SCI. The hallmark of treatment is range-of-motion to maintain joint function for activities such as sitting tolerance and transfers. Research has refuted the idea that active physical therapy may be a causative factor in the development of HO. ${ }^{4,5,8}$ NSAIDs have been found to be of benefit when used prophylactically for the prevention of $\mathrm{HO}$ or minimize its calcification in able-bodied patients following total joint replacement. ${ }^{18-20}$ The mechanism by which NSAIDs produce this effect is not well understood; antiprostaglandins may hinder pluripotential cells from differentiating into osteoblasts. ${ }^{19}$ The efficacy of NSAIDs in the prevention of HO in SCI has not been adequately studied. Diphosphonates, particularly etidronate disodium, have been shown to be of benefit in both the prevention and treatment of $\mathrm{HO}$ after surgical resection as well as in minimizing the amount of calcification in existing $\mathrm{HO}$ in SCI. ${ }^{3,20-22}$ Disphosphates are believed to inhibit the incorporation of calcium phosphate into crystalline hydroxyapatite. Warfarin has recently been described as providing some benefit in the prevention of $\mathrm{HO}^{23}$ The proposed mechanism of action is via inhibition of vitamin $\mathrm{K}$ dependent decarboxylation which is essential to the production of osteocalcin. Radiation therapy has been used successfully in preventing HO recurrence following total hip arthroplasty and as a primary treatment of $\mathrm{HO} .{ }^{24}$ The proposed mechanism of action is via the disruption of the differentiation process of pluripotential cells into osteoblasts. Postoperative radiation therapy has recently been reported to decrease the recurrence of $\mathrm{HO}$ after surgical resection in SCI patients. ${ }^{25}$ It should be noted that the maturity of the HO was not assessed preoperatively in this study.

Surgical resection of $\mathrm{HO}$ is undertaken in those patients in whom medical management has failed, resulting in a loss of function. Surgical complications are common and the risk of recurrence of $\mathrm{HO}$ is significant. ${ }^{4,7,17,25}$ It has long been felt that the resection of $\mathrm{HO}$ should not occur until the $\mathrm{HO}$ is mature. ${ }^{4,9,11,15,17,21}$ However, no adequate prospective controlled studies are available to confirm this concept. The exact timing of maturity is somewhat vague but traditionally felt to occur at 12 to 18 months after the development of $\mathrm{HO}^{4,6,9,11,20,22}$ Roentgenograms are unreliable indicators of maturity. 4,13,17,18,26 Interpretation of roentgenograms is difficult and immature components may be obscured by mature bone. The assessment of maturity via roentgenograms does not correlate well with alkaline phosphatase levels either. ${ }^{6}$ Alkaline phosphatase levels may remain elevated for years or may never return to normal. ${ }^{13,17,18}$ The most sensitive indicator for maturity of $\mathrm{HO}$ is felt to be the $99 \mathrm{~m}-\mathrm{Tc}-\mathrm{MDP}$ bone scan. ${ }^{13,16,17,27}$ A decreasing trend of activity on serial studies is felt to be indicative of maturing $\mathrm{HO}$ and a necessary requirement prior to surgical intervention. Bone scans may remain active for many years after the development of $\mathrm{HO}^{6}$ As well, bone scans, like roentgenograms, are subject to individual interpretation. $^{6,27}$ If surgery is delayed until the bone scan reveals nonactivity in the SCI patient, the risk of fracture secondary to disuse osteopenia may increase significantly. ${ }^{18}$ Even with evidence of maturation of HO via bone scan, postsurgical recurrence rates remain high., 13,17,18,27 Garland's study on resection of HO concluded that normal bone scans, alkaline phosphatase levels, and mature roentogenographic appearance of $\mathrm{HO}$ were unreliable predictors of recurrence. ${ }^{12}$ In studies of $\mathrm{HO}$ in SCI, radiographic evidence of maturity and normal alkaline phosphatase levels were of limited value in predicting recurrence. ${ }^{13,17,27}$ Garland and Orwin's prospective study found little correlation between maturity rating scales and the extent of recurrence. ${ }^{17}$ More importantly, Stover noted that the extent of recurrence was not 
found to be related to the length of onset of $\mathrm{HO}$ to surgery. ${ }^{18}$

Studies of $\mathrm{HO}$ associated with traumatic brain injury (TBI) have concluded that postoperative recurrence correlated with the severity of the neurologic lesion and not with the timing of surgery. ${ }^{28}$ Interestingly, surgical resection was undertaken despite continued activity on bone scans. No recurrence occurred postoperatively, even in those patients who underwent surgery at 5 months after the diagnosis of HO. In SCI, a complete neurologic lesion is felt to be a risk factor for the initial development of $\mathrm{HO}$ but has not been associated with its recurrence. ${ }^{6,14,17}$

Given the confounding nature of the literature related to the determination of maturity of $\mathrm{HO}$ and the appropriate timing for surgical intervention especially in light of the successful resection of immature HO in TBI and our case report, we suggest that surgery need not be delayed until complete maturity occurs. Further prospective study in the treatment of immature $\mathrm{HO}$ using combinations of surgical resection, NSAIDs, diphosphonates, and radiation therapy is warranted.

\section{References}

1 Dejerine M, Ceillier MA. Paraosteoarthropathies of paraplegic patients by spinal cord lesion. Ann Med 1918; 5: 497-535.

2 Venier L, Ditunno J. Heterotopic ossification in the paraplegic patient. Arch Phys Med Rehabil 1979; 52: 475-479.

3 Stover S, Hahn H, Miller J. Disodium etidronate in the prevention of heterotopic ossification following spinal cord injury. Paraplegia 1976; 14: 146-156.

4 Wharton G, Morgan T. Ankylosis in the paralyzed patient. $J$ Bone and Joint Surg 1970; 52A: 105-112.

5 Hardy A, Dickson J. Pathological ossification in traumatic paraplegia. J Bone and Joint Surg 1963; 45B: 76-87.

6 Tibone J, Sakimura I, Nickel V, Hsu J. Heterotopic ossification around the hip in spinal cord-injured patients. $J$ Bone and Joint Surg 1978; 60A: $769-775$.

7 Hsu J, Sakimura I, Stauffer E. Heterotopic ossification around the hip joint in spinal cord injured patients. Clin Orthop 1975; 112: $165-169$.

8 Stover S, Hataway C, Zeiger H. Heterotopic ossification in spinal cord injured patients. Arch Phys Med Rehabil 1975; 56: 199-204.

9 Wharton G. Heterotopic ossification. Clin Orthop 1975; 112: $142-149$.
10 Hernandez A, Forner J, De La Fuente T, et al. The para-articular ossifications on our paraplegics and tetraplegics: a survey of 704 patients. Paraplegia 1978; 16: $272-275$.

11 Chantraine A, Minaire P. Para-osteoarthropathies. Scand J Rehabil 1981; 13: 31-37.

12 Garland D. Clinical observations on fracture and heterotopic ossification in the spinal cord and brain injured populations. Clin Orthop 1988; 233: 86-101.

13 Tanaka T, Rossier A, Hussey R, et al. Quantitative assessment of para-osteo-arthropathy and it's maturation on serial radionuclide bone images. Radiology 1977; 123: 217-221.

14 Lal S, Hamilton B, Heinemann A, Betts H. Risk factors for heterotopic ossification in spinal cord injury. Arch Phys Med Rehabil 1989; 70: $387-390$.

15 Kewalramani L. Ectopic ossification. Am J Phys Med 1977; 56: $99-121$.

16 Freed J, Hahn H, Menter R, Dillon T. The use of three-phase bone scan in the early diagnosis of heterotopic ossification and in the evaluation of didronel therapy. Paraplegia 1982; 20: 208 216.

17 Garland D, Orwin J. Resection of heterotopic ossification in patients with spinal cord injuries. Clin Orthop 1989; 242: 169 176

18 Stover S, Niemann K, Tullous J. Experience with surgical resection of heterotopic ossification in spinal cord injury patients. Clin Orthop 1991; 263: 71-77.

19 Cella J, Salvati E, Sculco T. Indomethacin for the prevention of heterotopic ossification following total hip arthroplasty. $J$ Arthroplasty 1988; 3: 229-234.

20 Garland D. A clinical perspective on common forms of acquired heterotopic ossification. Clin Orthop 1990; 263: 13-29.

21 Subbarao J, Nemchasky B, Gratzer M. Resection of heterotopic ossification and didronel therapy-regaining wheelchair independence in the spinal cord injured patient. J Am Paraplegia Society 1987; 10: $3-7$.

22 Garland D, Alday B, Venos K, Vogt J. Disphosphonate treatment for heterotopic ossification in spinal cord injury patients. Clin Orthop 1983; 176: $197-200$.

23 Buschbacher R, McKinley W, Buschbacher L, et al. Warfarin in prevention of heterotopic ossification. Am J Phys Med \& Rehabil 1992; 71: 86-91.

24 Sautter-Bihl M, Libermeister E, Heize H, et al. The radiotherapy of heterotopic ossification in paraplegics. Strahlentherapie und Onkologie 1995; 171: 454-459.

25 Meiners T, Abel R, Bohm V, Gerner H. Resection of heterotopic ossification of the hip in spinal cord injured patients. Spinal Cord 1997; 35: 443 - 445.

26 Rossier A, Bussat P, Infane F, et al. Current facts on para-osteoarthropathy. Paraplegia 1973; 11: 36-78.

27 Muheim G, Donath A, Rossier A. Serial scintigrams in the course of ectopic bone formation in paraplegic patients. Am J Roentgenol 1973; 118: 865 -869.

28 Frischhut B, Stockhammer G, Saltuari L, et al. Early removal of periarticular ossifications in patients with head injury. Acta Neurologica 1992; 15: $114-122$. 